\title{
Experimental determination of electron inelastic mean free path of components in a magnetic read head
}

\author{
X. Xu, L. Vinh, J. Risner-Jamtgaard, and D. Yaney \\ Hitachi Global Storage Technologies, Inc., 5600 Cottle Road, San Jose, CA 95193
}

In today's disk drive industry, focused ion beam (FIB) tools are commonly used to prepare site specific TEM specimens from magnetic recording heads. However as device dimensions shrink, it becomes increasingly important to know the thickness of the TEM specimen in relation to device dimensions. To derive the sample thickness of a TEM specimen, a log-ratio method [1] is typically used, assisted by a software package such as Gatan DigitalMicrograph, and the value of the electron inelastic mean free path (IMFP) is acquired in the form of thickness/IMFP ( $t / \lambda)$. Most IMFP values are computed from parameterized equations with certain approximations [2]. Here, a combination of FIB and energy filtered imaging in the TEM is used to measure the specimen thickness directly. Then, this value is applied to the acquired relative thickness $(t / \lambda)$ map generated by the log-ratio method, and the IMFP values for various sample components are determined.

The TEM specimen is a tunneling magneto-resistance read (TMR) head. Surrounding the head is plated $\mathrm{Ni}_{80} \mathrm{Fe}_{20}$. Important layers within the head include, from bottom to top: 4$\mathrm{nm}$ Ta and 2-nm $\mathrm{NiFe}_{\mathrm{x}}$ seed layers, followed by a 7-nm anti-ferromagnetic layer, several $\mathrm{CoFe}_{\mathrm{x}}$ layers, a $\mathrm{MgO}$ barrier layer, and 6-nm $\mathrm{Ru}$ and 2-nm Ta cap layers. A FEI DualBeam 835 FIB was used to prepare the TEM sample. The area of interest was first coated in-situ with $\mathrm{SiO}_{x}$ and tungsten to protect the device. A cross-section segment containing the head was removed, micro-manipulated onto a $\mathrm{Cu}$ grid, and then thinned to electron transparency. A JEOL 2010F FEG TEM operated at $197 \mathrm{KeV}$ with a Gatan 863 Tridiem imaging filter was used to collect the relative thickness map (Fig. 1A). The map was acquired by the standard log-ratio method (Gatan DigitalMicrograph), and yielded a relative thickness value $(n)$ where $n=t / \lambda$. This relative thickness was obtained by averaging values from an area of $200 \times 1$ pixels to minimize artifacts from diffracting grains, and then plotted as a line profile (Fig. 1B).

To know the true thickness of the specimen, the TEM sample was then returned to the FIB, cut along the dashed line marked in the Fig. 1A, and thinned to electron transparency again. A bright field TEM image was then obtained, and the real thickness (t) was measured from the bright field image (Fig. 1B). Finally, the IMFPs of the layers were calculated $(\lambda=\mathrm{t} / \mathrm{n})$ and listed in Table 1 . High resolution and energy-filtered images indicate that the surface of the sample was damaged by FIB milling creating a $\sim 2 \mathrm{~nm}$ amorphous layer on each side. This amorphous damage layer was not considered in the measurement of $(\mathrm{t})$.

For $\mathrm{Ni}_{80} \mathrm{Fe}_{20}$, the thickness averages $26.7 \mathrm{~nm}$ based on three measurements, and the calculated IMFP of polycrystalline $\mathrm{Ni}_{80} \mathrm{Fe}_{20}$ is $77.6 \mathrm{~nm}$. It should be noted that the calculated IMFP values from the thin layers should consider the electrons interfering with 
the neighboring layers when scattered. However, these results provide useful information for comparing layers of similar physical dimension.

\section{References}

[1] R. F. Egerton, Electron Energy-Loss Spectroscopy in the Electron Microscope, $2^{\text {nd }}$ edition, Plenum Press, New York, 1996.

[2] D. B. Williams and C. B. Carter, Transmission Electron Microscopy, Plenum Press, New York, 1996.

[3] R. F. Egerton and S. C. Cheng, Ultramicroscopy (21), 1987
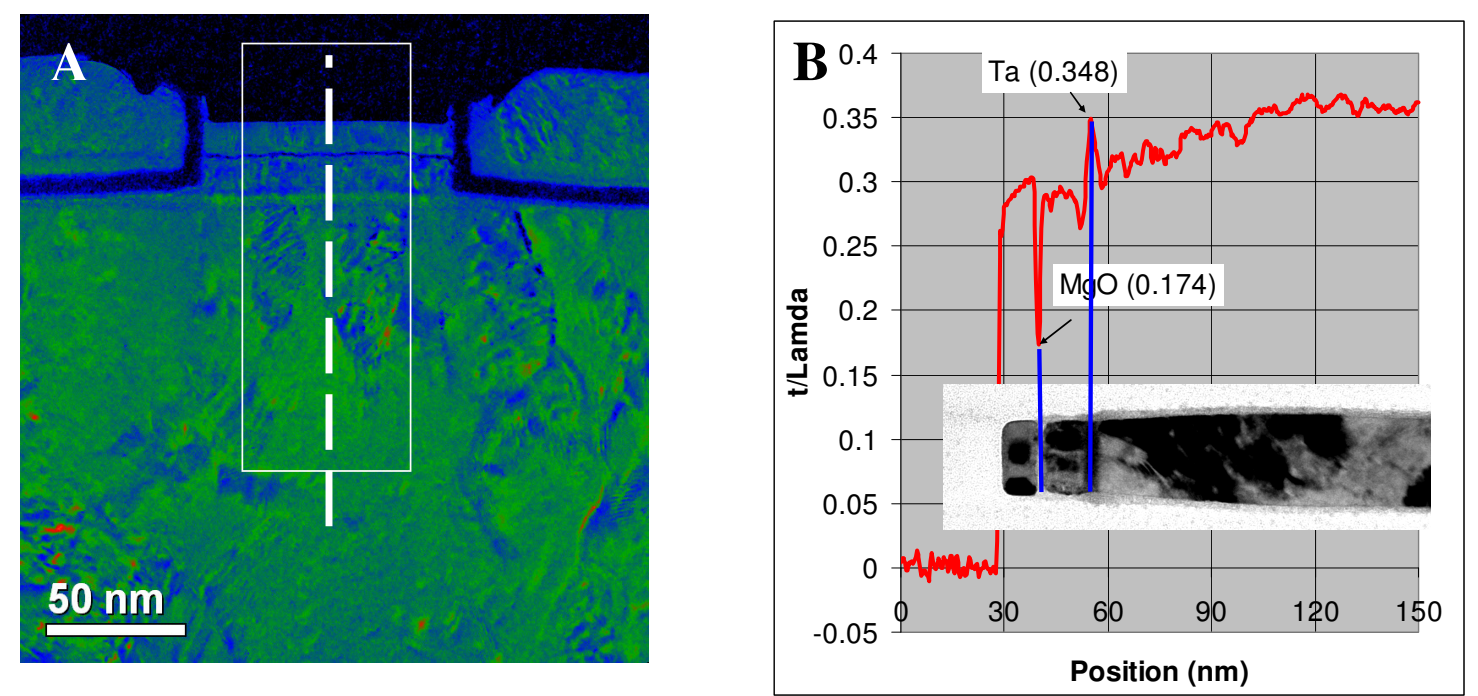

Fig. 1. A. Relative thickness map $(\mathrm{t} / \lambda)$ acquired by log-ratio calculations and shown in temperature scale. A $10 \mathrm{eV}$ energy window was used for collection. B. Line profile showing how the relative thickness (n) varies with the real thickness $(\mathrm{t})$, and $\mathrm{t}$ was measured from the corresponding bright field TEM image.

TABLE 1. IMFP $(\lambda)$ Values Calculated from Measured Values of Real Thickness $(t)$ and Relative Thickness $(\mathrm{t} / \lambda)$

\begin{tabular}{|c|c|c|c|c|}
\hline $\begin{array}{c}\text { Element/ } \\
\text { Compound }\end{array}$ & $\begin{array}{c}\text { Amorphous } \\
\text { /Crystalline }\end{array}$ & $\mathbf{t}(\mathbf{n m})$ & $\mathbf{n = t} / \boldsymbol{\lambda}$ & $\boldsymbol{\lambda}(\mathbf{n m})$ \\
\hline $\mathrm{Ru}$ & Crystalline & 22.8 & 0.289 & $\mathbf{7 8 . 9}$ \\
\hline $\mathrm{MgO}$ & Crystalline & 22.4 & 0.174 & $\mathbf{1 2 8 . 7}$ \\
\hline $\mathrm{Ta}$ & Amorphous & 21.7 & 0.348 & $\mathbf{6 2 . 3}$ \\
\hline $\mathrm{Ni}_{80} \mathrm{Fe}_{20}$ & Crystalline & 25.1 & 0.321 & $\mathbf{7 8 . 2}$ \\
\hline $\mathrm{Ni}_{80} \mathrm{Fe}_{20}$ & Crystalline & 27.7 & 0.358 & $\mathbf{7 7 . 4}$ \\
\hline $\mathrm{Ni}_{80} \mathrm{Fe}_{20}$ & Crystalline & 27.4 & 0.355 & $\mathbf{7 7 . 2}$ \\
\hline
\end{tabular}

